\title{
ANTI-PERIODIC BOUNDARY VALUE PROBLEM FOR NONLINEAR FIRST ORDER ORDINARY DIFFERENTIAL EQUATIONS
}

\author{
DANIEl Franco, JUAN J. NiETO AND DONAL O'REGAN
}

\begin{abstract}
We prove several new existence results for a nonlinear anti-periodic first order problem using a Leray-Schauder alternative. Two definitions of lower and upper solutions are presented and we show in this paper the validity of the lower and upper solution method. Also, we give a method to generate a sequence of approximate solutions converging to a solution of the anti-periodic problem.
\end{abstract}

\section{Mathematics subject classification (2000): 34B15, 34C25.}

Key words and phrases: Anti-periodic boundary value problem, Leray-Schauder alternative, upper and lower solutions.

\section{REFERENCES}

[1] A. Abdurrahman, F. Anton, J. Bordes, Half-string oscillator approach to string field theory (Ghost sector. I), Nuclear Phys. B, 397 (1993), 260-282.

[2] R. Aftabizadeh, Y. K. Huang, N. H. Pavel, Nonlinear third-order differential equations with antiperiodic boundary conditions and some optimal control problems, J. Math. Anal. Appl., 192 (1995), 266-293.

[3] R. Aftabizadeh, N. H. Pavel, Y. K. Huang, Anti-periodic oscillations of some second-order differential equations and optimal control problems, J. Comput. Appl. Math., 52 (1994), 3-21.

[4] C. AHn, C. Rim, Boundary flows in general coset theories, J. Phys. A, 32 (1999), 2509-2525.

[5] S. Aizicovici, S. McKibben, S. ReICH, Anti-periodic solutions to nonmonotone evolution equations with discontinuous nonlinearities, Nonlinear Anal. Ser. A: Theory, Methods, 43 (2001), 233-251.

[6] S. Aizicovici, N. H. PAvel, Anti-periodic solutions to a class of nonlinear differential equations in Hilbert space, J. Funct. Anal., 99 (1991), 387-408.

[7] S. AizICOVICI, S. ReICH, Anti-periodic solutions to a class of non-monotone evolution equations, Discrete Contin. Dynam. Systems, 5 (1999), 35-42.

[8] A. CABADA, J. J. NIETO, Fixed points and approximate solutions for nonlinear operator equations, J. Comput. Appl. Math., 113 (1999), 17-25.

[9] H. L. Chen, Antiperiodic wavelets, J. Comput. Math., 14 (1996), 32-39.

[10] Y. Q. CHEN, On Massera's theorem for anti-periodic solution, Adv. Math. Sci. Appl., 9 (1999), 125-128.

[11] F. J. DELVOS, L. KNOCHE, Lacunary interpolation by antiperiodic trigonometric polynomials, BIT, 39 (1999), 439-450.

[12] D. FrANCO, J. J. NIETO, First-order impulsive ordinary differential equations with anti-periodic and nonlinear boundary conditions, Nonlinear Anal., 42 (2000), 163-173.

[13] H. KleINERT, A. CHERVYAKOV, Functional determinants from Wronski Green functions, J. Math. Phys., 40 (1999), 6044-6051.

[14] G. S. Ladde, V. Lakshmikantham, A. S. Vatsala, Monotone Iterative Techniques for Nonlinear Differential Equations, Pitman, Boston, (1985).

[15] M. NAKAO, Existence of an anti-periodic solution for the quasilinear wave equation with viscosity, J. Math. Anal. Appl., 204 (1996), 754-764.

[16] H. OKосHI, On the existence of anti-periodic solutions to a nonlinear evolution equation associated with odd subdifferential operators, J. Funct. Anal., 91 (1990), 246-258. 
[17] D. O'Regan, Existence Theory for Nonlinear Ordinary Differential Equations, Kluwer, Dordrecht, (1997).

[18] S. Pinsky, U. TRITTMAnN, Antiperiodic boundary conditions in supersymmetric discrete light cone quantization, Phys. Rev. D (3), 62 (2000), 87701, 4 pp.

[19] P. SOUPLET, Optimal uniqueness condition for the antiperiodic solutions of some nonlinear parabolic equations, Nonlinear Anal., 32 (1998), 279-286.

[20] Y. YIN, Anti-periodic solutions of some semilinear parabolic boundary value problems, Dynam. Contin. Discrete Impuls. Systems 1 (1995), 283-297.

[21] Y. YIN, Monotone iterative technique and quasilinearization for some anti-periodic problems, Nonlinear World, 3 (1996), 253-266.

[22] Y. YIN, Remarks on first order differential equations with anti-periodic boundary conditions, Nonlinear Times Digest, 2 (1995), 83-94. 